\title{
LOCALIZACION DE P-BRANAS EN SUPERGRAVEDAD Y TEORIA-M
}

\author{
Fulgencio Villegas Silva ${ }^{\text {a }}$ \\ ${ }^{a}$ Departamento de Física Atómica y Nuclear, Facultad de Ciencias Físicas, Universidad Nacional Mayor de San Marcos. \\ Ciudad Universitaria Av. Venezuela Cuadra 34. Apartado. Postal 14-0149, Lima 14, Perú.
}

\begin{abstract}
Resumen
Se analizan los resultados recientes referentes a una clase especial de soluciones tipo p-branas a la teoría de supergravedad en once y diez dimensiones. Se discute la no localización y localización de las intersecciones de las p-branas. Se presenta el nuevo mecanismo de "ennegrecimiento" de branas localizadas.
\end{abstract}

Palabras claves: P-branas, Supercuerdas, Supergravedad, Dimensiones extra.

\begin{abstract}
Recent results concerning a special class of p-brane solutions in eleven-dimensional and ten-dimensional supergravities are reviewed. Delocalised as well as localized intersections of p-branes are discussed. The new mechanism of "Blackening" localized branes is presented.
\end{abstract}

Keywords: P-branes, Superstring, Supergravity, Extra dimensions.

\section{Introducción}

Las soluciones tipo p-branas a la teoría de la supergravedad son relevantes para diferentes aspectos de la teoría de supercuerdas, relatividad general y teorías de gauge supersimétricas. Las p-branas son una manifestación de baja energía de la teoría de cuerdas no-perturbativa [1].Es determinante el rol especial que cumple el estudio de las p-branas en el reciente desarrollo de la teoría de cuerdas. Las p-branas se establecen como soluciones de la teoría de supergravedad y pueden ser investigadas por métodos clásicos, ellas proporcionan un camino para obtener información no-perturbativa acerca de la teoría cuántica de cuerdas vía cálculos clásicos. Las p-branas también son importantes por la existencia de dualidades que conectan las diferentes teorías de supercuerdas y apoyan la conjetura de que los cinco tipos de supercuerdas conocidas son puntos diferentes de una única teoría llamada teoría-M [2].
Podrían inspeccionarse fiablemente las dualidades en el nivel clásico y las diferentes conexiones entre p-branas las cuales proporcionan las herramientas necesarias para ello. Es difícil de sobrestimar el papel de las pbranas en la física del agujero negro. Uno de los recientes logros más importantes aquí es una interpretación microscópica de la entropía de los agujeros negros [3]. Los agujeros negros son considerados como configuraciones de branas envueltas y teniendo en cuenta los micros estados de las branas permite derivar la fórmula de Bekenstein-Hawking. Recientemente una nueva aplicación de las p-branas fue encontrada. Ello parecía ser importante para la interpretación de resultados no-perturbativos en teorías gauge supersimétricas vía la teoría-M [4]. De esta manera los aspectos diferentes de la dinámica no-perturbativa tienen una explicación geométrica clara en lo que se refiere a las configuraciones de las branas. 


\section{Soluciones tipo p-branas}

Considerando las p-branas en supergravedad con once dimensiones. El sector bosónico de esta teoría consiste de una métrica y un potencial 3forma. La parte correspondiente a la acción es [5]

$S=\frac{1}{2 \kappa^{2}} \int d^{11} x \sqrt{-G}\left(R-\frac{1}{2.4 !} F_{M N P Q}^{2}\right)-\frac{1}{12 \kappa^{2}} \int A \wedge F \wedge F$.

Las p-branas son soluciones puramente bosonicas, así el sector fermiónico de la teoría no es importante en este trabajo. La supergravedad en once dimensiones admite dos soluciones básicas, las cuales corresponden a la M2-brana y a la M5-brana de la teoría-M. La solución M2-brana tiene la siguiente forma [6]

$d s^{2}=H^{1 / 3}\left(H^{-1}\left(-d t^{2}+d y_{1}^{2}+d y_{2}^{2}\right)+\left(d x_{1}^{2}+\ldots+d x_{8}^{2}\right)\right)$,

$$
A_{t y_{1} y_{2}}={ }^{2} \underset{t y_{1} y_{2}}{ } H^{-1}
$$

donde $H$ es la función Harmónica, la cual depende de $x$-coordenadas

$$
H=1+\frac{Q}{r^{6}}, \quad r=|\vec{r}| \text {. }
$$

Esta solución describe una sola membrana con un volumen-mundo orientado a lo largo de $t, y_{1}$, $y_{2}$ y localizada en el punto $\mathrm{r}=0$ en el espacio transversal. La M2-brana transporta la carga 4forma eléctrica la cual es definida como la integral e la dual 7-forma alrededor a una 7esfera que rodea la brana. La M2-brana es un estado BPS, preserva $1 / 2$ de la supersimetría inicial. Una importante solución de la supergravedad en once dimensiones es la M5brana [7]

$d s^{2}=H^{2 / 3}\left(H^{-1}\left(-d t^{2}+d y_{1}^{2}+\ldots d y_{5}^{2}\right)+\left(d x_{1}^{2}+\ldots+d x_{5}^{2}\right)\right)$,

$$
F_{\alpha_{1} \ldots \alpha_{4}}={ }^{2}{ }_{\alpha_{1} \ldots \alpha_{5}} \partial_{\alpha_{5}} H, \quad H=1+\frac{Q}{r^{6}} .
$$

La M5-brana transporta caga 4-forma magnética la cual es obtenida por integración de $\mathrm{F}$ alrededor de una 4-esfera que rodea la M5-brana. La M2-brana y la M5-brana también preservan un medio de la supersimetría. A veces se dice que una solución brana cuya función armónica es independiente de varias coordenadas transversales es "no-localizada". Esto significa que uno no puede determinar la posición de la brana en algunas direcciones, Aparece la incertidumbre en la localización.

\section{Intersección de branas}

Las soluciones presentadas son los bloques para las construcciones de configuraciones más complicadas que corresponden a intersecciones de branas. Como un ejemplo de intersección no localizada se puede considerar una configuración (3|M 5, M 5) de dos M5-branas intersecándose en una 3-brana $[8,9]$.

$$
\begin{gathered}
d s^{2}=H_{1}^{2 / 3}(x) H_{2}^{2 / 3}(x)\left(H_{1}^{-1}(x) H_{2}^{-1}(x)\left(-d t^{2}+d u_{1}^{2}+d u_{2}^{2}+d u_{3}^{2}\right)\right. \\
\left.+H_{1}^{-1}(x)\left(d y_{1}^{2}+d y_{2}^{2}\right)+H_{2}^{-1}(x)\left(d z_{1}^{2}+d z_{2}^{2}\right)+d x_{\alpha} d x^{\alpha}\right), \\
F_{z_{1} z_{2} \alpha \beta}={ }_{\alpha \beta \gamma}^{2} \partial_{\gamma} H_{1}(x), \quad F_{y_{1} y_{2} \alpha \beta}={ }^{2} \underset{\alpha \beta \gamma}{ } \partial_{\gamma} H_{2}(x)
\end{gathered}
$$

La primera brana es localizada en la dirección $t$, $u$, $y$, la segunda en la dirección $t, u, z$. Las coordenadas $y, z$ son llamadas transversas relativas. La importancia de esta solución es que la función armónica $\mathrm{H}_{1}(\mathrm{x})$ y $\mathrm{H}_{2}(\mathrm{x})$ depende del conjunto de coordenadas transversales las cuales son ortogonales a ambas branas. Significa que cada brana no es localizada en las direcciones transversales relativas. Excepto esta configuración existe otro par de intersecciones no localizadas que involucran M2-brana y M5brana, como son $[9,10]$

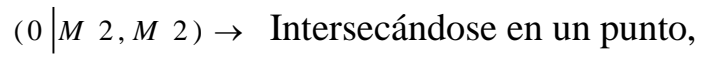

$(1 \mid M 2, M 5) \rightarrow$ M2-brana interseca a M5-brana a lo largo de 1-brana.

Notar que la intersección de branas es controlada por la regla general de intersección $[9,10]$. Esta regla de intersección es consistente con las dualidades [11]. Ahora analizaré las intersecciones localizadas de M-branas. Existen sólo dos configuraciones de este tipo. Uno de ellos describe dos M5-branas que se intersecan 
en una cuerda $(1 \mid M 5, M 5)$, la otra puede obtenerse agregando la tercera M2 - brana (1 $\mid M 5, M 5, M 2)$. El elemento de la línea y las componentes non-triviales de la forma diferencial para localizar la intersección de la M5-brana es

$$
\begin{aligned}
d s^{2}= & H_{1}^{2 / 3}(y) H_{2}^{2 / 3}(x)\left(H_{1}^{-1}(y) H_{2}^{-1}(x)\left(-d t^{2}+d u^{2}\right)\right. \\
& \left.+H_{1}^{-1}(y) d \vec{x}^{2}+H_{2}^{-1}(x) d \vec{y}^{2}+d \omega^{2}\right),
\end{aligned}
$$$$
F_{y_{i} y_{k} y_{t}, \omega}={ }^{2}{ }_{y_{i} y_{k} y_{i} y_{n}} \partial_{y_{n}} H_{1}(y), \quad F_{x_{i} x_{k} x_{i}, \omega}={ }^{2}{ }_{x_{i} x_{k} x_{t} x_{n}} \partial_{x_{m}} H_{2}(x) .
$$

Esta solución describe la M5-brana la cual es orientada a lo largo de las direcciones $\mathrm{t}, \mathrm{u}, \mathrm{y}$ e interfecta a la otra M5-brana localizada en la dirección $t, u, x$. Hay una diferencia importante entre estas soluciones y aquellas ya discutidas: Las funciones armónicas son ahora independientes del conjunto de direcciones transversales y solamente dependen de las direcciones transversales relativas. Esto es, la M5-brana se localiza ahora entre las direcciones tangentes de la otra M5 -brana pero es no localizada en la dirección transversa global que los separa. Notar que esta es también la diferencia en las reglas de intersección entre intersecciones no localizadas y localizadas de Mbranas $[12,13,14]$.

\section{Proceso de ennegrecimiento de branas}

Todas las soluciones discutidas anteriormente son BPS-saturadas. Además de ello uno puede considerar soluciones no-extremales que pueden interpretarse como las deformaciones de las extremales. El procedimiento para obtener soluciones no extremales de las extremales se denomina proceso de "ennegrecimiento". En el caso de una brana o no localización de la intersección de branas este procedimiento consiste en reemplazar en el espacio transversal global D-dimensional de los elementos de línea así como en la parte temporal de el $[15,16]$

$$
\begin{gathered}
d x_{\alpha} d x^{\alpha} \rightarrow f^{-1}(r) d r^{2}+r^{2} d \Omega_{D-1}, \\
d t^{2} \rightarrow f(r) d t^{2},
\end{gathered}
$$

donde

$$
\begin{aligned}
& f(r)=1-\frac{\mu}{r^{D-2}} . \\
& A \rightarrow \sqrt{1+\frac{\mu}{Q}} .
\end{aligned}
$$

Como un ejemplo presentamos la intersección de una M5-brana no extremal obtenida de esta manera

$$
\begin{aligned}
d s^{2}= & H_{1}^{2 / 3}(x) H_{2}^{2 / 3}(x)\left(H_{1}^{-1}(x) H_{2}^{-1}(x)\left(-f(r) d t^{2}+d u_{1}^{2}+d u_{2}^{2}+d u_{3}^{2}\right)\right. \\
& \left.+H_{1}^{-1}(x)\left(d y_{1}^{2}+d y_{2}^{2}\right)+H_{2}^{-1}(x)\left(d z_{1}^{2}+d z_{2}^{2}\right)+f^{-1}(r) d r^{2}+r^{2} d \Omega_{2}\right),
\end{aligned}
$$

Notar que en límite $\mu \rightarrow 0$ se consigue la configuración extremal discutida. Otro límite $Q \rightarrow 0$ da un análogo multidimensional de la solución de Schwarzschild. Desafortunadamente este procedimiento no trabaja propiamente para las intersecciones localizadas de branas. Esta aplicación no extremiza solamente una brana, sino ambas. Se necesita otra aproximación que sea una generalización de este método. Fue propuesto [14] que el nuevo procedimiento de "ennegrecimiento" sea basado en deformaciones que en el límite del vacío no den la solución de Schwarzschild pero den soluciones más complicadas. Este procedimiento se aplicó a la intersección localizada de M5-branas presentando la configuración siguiente

$$
\begin{aligned}
d s^{2}= & H_{x}^{2 / 3} H_{y}^{2 / 3}\left(H_{x}^{-1} H_{y}^{-1}\left(-f_{x} f_{y} d t^{2}+d u^{2}\right)+H_{y}^{-1} f_{x}^{1 / 3}\left(f_{x}^{-1} d r_{x}^{2}+r_{x}^{2} d \Omega_{3}\right)\right. \\
& \left.+H_{x}^{-1} f_{x}^{1 / 3}\left(f_{y}^{-1} d r_{y}^{2}+r_{y}^{2} d \Omega_{3}\right)+f_{x}^{-2 / 3} d \omega^{2}\right),
\end{aligned}
$$

$H_{x}=1+\frac{Q_{x}}{r_{x}^{2}}, H_{y}=1+\frac{Q_{y}}{r_{y}^{2}}, f_{x}=1-\frac{\mu_{x}}{r_{x}^{2}}, f_{y}=1-\frac{\mu_{y}}{r_{y}^{2}}$.

El límite $\mu_{x}, \mu_{y} \rightarrow 0$ se retorna a la configuración extrema al anterior, el límite $Q_{x}, Q_{y} \rightarrow 0$ lleva a la nueva solución en el vacío de la supergravitación en once-dimensiones que difiere de la solución de Schwarzschild. 


\section{Supergravedad IIA}

Ahora se discute brevemente el tipo de supergravedad IIA. La acción de la supergravedad tipo IIA en diez dimensiones se obtiene por reducción dimensional en un círculo de supergravedad en once-dimensiones.

$$
\begin{aligned}
& S=\frac{1}{2 \kappa^{2}} \int d^{10} x \sqrt{-G}\left(R-\frac{1}{2}(\partial \phi)^{2}-\frac{1}{2.3 !} e^{-\phi} F_{3}{ }^{2}\right) \\
& \left.\frac{1}{2.2 !} e^{\frac{3 \phi}{2}} F_{2}{ }^{2}-\frac{1}{2.4 !} e^{\frac{\phi}{2}} F_{4}{ }^{2}\right)+(\text { Chern }- \text { Simons })+(\text { fermiones }),
\end{aligned}
$$

$$
F_{4}^{\prime}=d A_{3}+A_{1} \wedge F_{3} .
$$

La parte bosónica de la acción contiene los campos que vienen de los sectores NS-NS y R$\mathrm{R}$ de la supercuerda. El dilatón, la métrica $G_{M N}$ y la 2-forma $\mathrm{A}_{2}$ son campos NS-NS, la 1-forma $\mathrm{A}_{1}$ y 3-forma $\mathrm{A}_{3}$ son campos R-R. Los objetos multidimensionales que conducen cagas R-R son llamados D-branas. También se tiene NS-branas las cuales conducen cargas NS. De acuerdo con el campo contenido tenemos las siguientes branas

NS-Brana: NS1 (cuerda fundamental), NS\% (5-brana solitónica)

D-branas : $\quad$ D0, D2, D4, D6.

Así como en el caso de M-branas se construye las configuraciones de intersección de estas branas así también se puede considerar las deformaciones no extremales de ellas. Se presenta como ejemplo la configuración localizada de dos NS5-branas no extremales intersecándose en una cuerda [14]

$$
\begin{aligned}
d s^{2}= & H_{x}^{3 / 4} H_{y}^{3 / 4}\left(H_{x}^{-1} H_{y}^{-1} f_{x}^{-3 / 4}\left(-f_{x} f_{y} d t^{2}+d u^{2}\right)+H_{y}^{-1} f_{x}^{1 / 4}\left(f_{x}^{-1} d r_{x}^{2}+r_{x}^{2} d \Omega_{3}\right)\right. \\
& \left.+H_{x}^{-1} f_{x}^{1 / 4}\left(f_{y}^{-1} d r_{y}^{2}+r_{y}^{2} d \Omega_{3}\right)\right),
\end{aligned}
$$

$$
e^{-\phi}=H_{x}^{1 / 2} H_{y}^{1 / 2} f_{x}^{-1 / 2}
$$

$$
H_{x}=1+\frac{Q_{x}}{r_{x}^{2}}, H_{y}=1+\frac{Q_{y}}{r_{y}^{2}}, f_{x}=1-\frac{\mu_{x}}{r_{x}^{2}}, f_{y}=1-\frac{\mu_{y}}{r_{y}^{2}} .
$$

\section{Conclusiones}

En la conclusión, se ha discutido algunos problemas y preguntas abiertas acerca de las soluciones tipo p-branas en varias supergravedades. Se localizó la intersección de tres branas extremales (M5, M5 y M2 por ejemplo). Sin embargo en el caso de branas noextremales se mostró un par de intersecciones localizadas. Es interesante notar que es posible agregar una tercer brana non-extremal que se localiza en las direcciones transversales relativas. Uno de los problemas no resuelto es el de determinar soluciones a la supergravedad que representen branas que terminen en otras branas [4]. La importancia de tales soluciones es determinada por la posibilidad de interpretar algunos resultados en teorías gauge supersimétricas vía p-branas. Podría ser que estudiando estas configuraciones nos llevarán a entender más profundamente el fenómeno noperturbativo en la teoría de campos supersimétrica así como en la no-supersimétrica

\section{Referencias}

[1] E. Witten, Nucl. Phys. B443 (1995) 85.

[2] C. M. Hull and P.K. Townsend, Nuck.Phys. B438 (1995) 109

J. H. Schwarz, Phys.Lett.B367 (1996) 97.

Dinitri V. Gal'tsov, José P. S. Lemos, Gerard Clement, Supergravity p-branes, hep-th/ 0403112.

[3] Gulio Bonelli, Maxian Zabzine, From current algebras for p-branes to topological M-theory, hep-th/ 0507051.
A. Strominger and
C. Vafa, Phys. Lett. B379 (1996) 99.

C.G. Callan and J. M. Maldacena, Nucl. Phys. B472 (1996) 591.

[4] A. Hanany and E. Witten, Nucl. Phys. B492 (1997) 152. 
E. Witten, Nucl. Phys. B500 (1997) 3.

C. Gomez, R. Hernandez, Fields, strings and branes, hep-th/ 9711102.

A. Giveon, D. Kutasov, Brane Dynamics and Gauge Theory, hepth/ 9802067.

[5] Chiang-Mei Chen, Dinitri V. Gal'tsov, Nobugoshi ohta, Intersecting non-extreme p-branes on linear dilaton background, hep-th/ 056216.

E. Cremmer, B. Julia and J. Scherk, Phys. Lett. B76 (1978) 409.

[6] D. J. Duff and K.S. Stelle, Phys. Lett. B253 (1991) 113.

[7] Fulgencio Villegas Silva, Soluciones a la teoría de supergravedad utilizando objetos extendidos tipo p-branas, tesis de Maestría, UNMSM, Lima-Perú, 2005.

[8] G. Papadopoulos and P. K. Townsend, Phys. Lett. B380 (1996) 273.

[9] A. A. Tseytlin, Nucl. Phys. B475 (1996) 149.

[10] I. Ya. Aref'eva and O.A. Rytchkov, Incidence Matrix Description of intersecting p-brane Solutions, hepth/9612236.
[11] I. Ya. Aref'eva, M.G. Ivanov and O.A. Rytchkov, Propietes of Intersecting $p$ branes in Various Dimensions, Proceedings of the International Seminar " Supersymmetry and Quantum Field Theory".

[12] J. P. Gauntlett, D. A. Kastor and J. Traschen, Nucl. Phys. B478 (1996) 544.

[13] J. D: Edelstein, L. Tataru and R. Tatar, Rules for Localised Overlappings and Intersections of p-Branes, hep-th/9801049.

[14] I. Ya. Aref'eva, M.G. Ivanov , O.A. Rytchkov and I. V. Volovich, hepth/9802163.

[15] M. Cvetic and A.A. Tseytlin, Nucl. Phys. B478 (1996) 181.

[16] I. Ya. Aref'eva, M.G. Ivanov, and I. V. Volovich, Phys. Lett. B406 (1997) 44.

[17] M. G. Ivanov, p-brane solutions and Beltrami-Laplace operator, hepth/9710110. 